\title{
Model-based Statistical Tracking and Decision Making for Collision Avoidance Application
}

\author{
Rickard Karlsson, Jonas Jansson, Fredrik Gustafsson \\ Control \& Communication \\ Department of Electrical Engineering \\ Linköpings universitet, SE-581 83 Linköping, Sweden \\ WWW: http://Www. control.isy.liu.se \\ E-mail: rickard@isy.liu.se, jansson@isy.liu.se \\ fredrik@isy.liu.se \\ 4th March
}

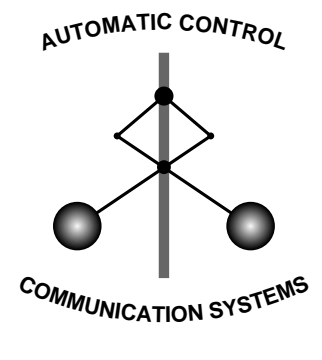

LINKÖPING

\author{
Report no.: LiTH-ISY-R-2599
}

Submitted to ACC 2004

Technical reports from the Control \& Communication group in Linköping are available at http://www.control.isy.liu.se/publications. 


\begin{abstract}
A growing research topic within the automotive industry is active safety systems. These systems aim at helping the driver avoid or mitigate the consequences of an accident. In this paper a collision mitigation system that performs late braking is discussed. The brake decision is based on estimates from tracking sensors. We use a Bayesian approach, implementing an extended Kalman filter (EKF) and a particle filter to solve the tracking problem. The two filters are compared for different sensor noise distributions in a Monte Carlo simulation study. In particular a bi-modal Gaussian distribution is proposed to model measurement noise for normal driving. For ideal test conditions the noise probability density is derived from experimental data. The brake decision is based on a statistical hypothesis test, where collision risk is measured in terms of required acceleration to avoid collision. The particle filter method handles this test easily. Since the test is not analytically solvable a stochastic integration is performed for the EKF method. Both systems perform well in the simulation study under the assumed sensor accuracy. The particle filter based algorithm is also implemented in a real-time testbed and fullfilled the on-line requirements.
\end{abstract}

Keywords: Particle filter, Bayesian estimation, Collision Avoidance, Hypothesis Testing 


Avdelning, Institution
Division, Department
Control \& Communication
Department of Electrical Engineering

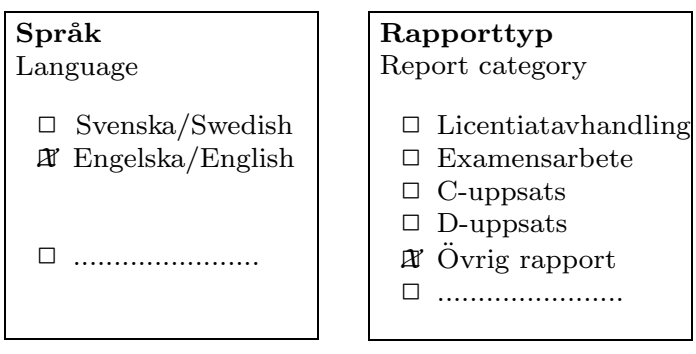

URL för elektronisk version

http://www.control.isy.liu.se

\begin{tabular}{|c|c|}
\hline $\begin{array}{l}\text { ISBN } \\
\ldots \ldots \ldots \ldots \\
\text { ISRN }\end{array}$ & .... \\
\hline $\begin{array}{l}\text { Serietitel och serienummer } \\
\text { Title of series, numbering }\end{array}$ & ISSN \\
\hline LiTH-ISY-R-2599 & \\
\hline
\end{tabular}

Titel

Title Model-based Statistical Tracking and Decision Making for Collision Avoidance Application

Författare

Author

Rickard Karlsson, Jonas Jansson, Fredrik Gustafsson,

\begin{abstract}
Sammanfattning
Abstract

A growing research topic within the automotive industry is active safety systems. These systems aim at helping the driver avoid or mitigate the consequences of an accident. In this paper a collision mitigation system that performs late braking is discussed. The brake decision is based on estimates from tracking sensors. We use a Bayesian approach, implementing an extended Kalman filter (EKF) and a particle filter to solve the tracking problem. The two filters are compared for different sensor noise distributions in a Monte Carlo simulation study. In particular a bi-modal Gaussian distribution is proposed to model measurement noise for normal driving. For ideal test conditions the noise probability density is derived from experimental data. The brake decision is based on a statistical hypothesis test, where collision risk is measured in terms of required acceleration to avoid collision. The particle filter method handles this test easily. Since the test is not analytically solvable a stochastic integration is performed for the EKF method. Both systems perform well in the simulation study under the assumed sensor accuracy. The particle filter based algorithm is also implemented in a real-time testbed and fullfilled the on-line requirements..
\end{abstract}

\title{
Nyckelord
}

Keywords

Particle filter, Bayesian estimation, Collision Avoidance, Hypothesis Testing 


\section{Model-based Statistical Tracking and Decision Making for Collision Avoidance Application}

\author{
Rickard Karlsson \\ Control \& Communication \\ Linköping University \\ SE-58183 Linköping, Sweden \\ rickardeisy.liu.se
}

\author{
Jonas Jansson \\ Volvo Car/Control \& Communication \\ Active Safety \\ SE-405 31 Göteborg, Sweden \\ jjanssol@volvocar.com
}

\author{
Fredrik Gustafsson \\ Control \& Communication \\ Linköping University \\ SE-58183 Linköping, Sweden \\ fredrikeisy.liu.se
}

\begin{abstract}
A growing research topic within the automotive industry is active safety systems. These systems aim at helping the driver avoid or mitigate the consequences of an accident. In this paper a collision mitigation system that performs late braking is discussed. The brake decision is based on estimates from tracking sensors. We use a Bayesian approach, implementing an extended Kalman filter (EKF) and a particle filter to solve the tracking problem. The two filters are compared for different sensor noise distributions in a Monte Carlo simulation study. In particular a bi-modal Gaussian distribution is proposed to model measurement noise for normal driving. For ideal test conditions the noise probability density is derived from experimental data. The brake decision is based on a statistical hypothesis test, where collision risk is measured in terms of required acceleration to avoid collision. The particle filter method handles this test easily. Since the test is not analytically solvable a stochastic integration is performed for the EKF method. Both systems perform well in the simulation study under the assumed sensor accuracy. The particle filter based algorithm is also implemented in a real-time testbed and fullfilled the on-line requirements.
\end{abstract}

\section{INTRODUCTION}

A current trend in automotive industry is to introduce active safety systems that avoid or mitigate collisions. One system with a potential large positive impact on accident statistics is forward collision avoidance systems (FCAS), using sensors such as radar, lidar and cameras to monitor the region in front of the vehicle. A tracking algorithm is used to estimate the state of the objects ahead and a decision algorithm uses the estimated states to determine any action. We will specifically look at a system that performs late braking to reduce the collision speed, which is referred to as collision mitigation by braking system (CMbBS). This type of system is discussed further in [1]. There are several motivations for this kind of system. One is its potential ability to affect rear-end collisions which constitute approximately $30 \%$, see [2], of all collisions. Furthermore human factors contribute to approximately $90 \%$ of all traffic accidents, [3]. For reasons such as driver acceptance and legal requirements of the system, the tracking and decision algorithms are crucial. Current state of the art automotive tracking algorithms use Kalman or extended Kalman filter (EKF) due to computational cost.

In this paper we will propose a new method for collision avoidance applications based on Bayesian estimation methods and hypothesis testing. Two tracking algortihms are tested. One based on EKF an one on the particle filter. The latter method is also implemented in a real-time test system and its real-time feasability is demonstrated in spite of its computer intensive complexity compared to the EKF.

\section{TRACKIng AND Vehicle Models}

\section{A. Tracking model}

The general tracking model takes the form of a nonlinear state space model for the vehicle dynamics and sensor measurements

$$
\begin{aligned}
x_{t+1} & =f\left(x_{t}, u_{t}, w_{t}\right), \\
y_{t} & =h\left(x_{t}\right)+e_{t} .
\end{aligned}
$$

For our radar based CMbB system, the state vector, $x_{t} \in$ $\mathbb{R}^{n}$, contains relative position to the other vehicle of potential collision risk, and relative velocity. Here both longitudinal and lateral directions are used. Further, $u_{t}$ is the known inputs from the accelerator, brake and steering wheel. These are also present for the target vehicle, but then un-measureable and hence treated as state noise, $w_{t}$. The measurement relation (1b) comes from radar measurements of range, range rate and bearing. The measurement noise, $e_{t}$, includes clutter, multi-path and multiple reflection points in the vehicle ahead.

\section{B. Estimation approaches}

For the $\mathrm{CMbB}$ system the measurement noise and the process noise (driver inputs) are not necessarily Gaussian. Thus we need a recursive estimation method that can handle this. In this paper we will study a general non-linear and non-Gaussian Bayesian estimation problem. This is in general non-tractable, but using the particle filter method [4] (see Section IV-A) a recursive solution to the problem is given. We will also compare with the linearized solution using the extended Kalman filter (EKF).

\section{State noise model}

The classical assumption in target tracking is to assume a Gaussian distribution for $w_{t}$. In Fig 1 recorded driving data provided by Volvo is used to make histograms of the longitudinal component of the acceleration noise, $w_{t}$. The data is of course highly correlated in time, since the acceleration does not fluctate rapidly. It could be used by introducing a Markov chain based on the empirical 
data. However, for braking situations probably a distribution based on accelerations collected close to rapid decelerations should be used instead. A further and natural option is

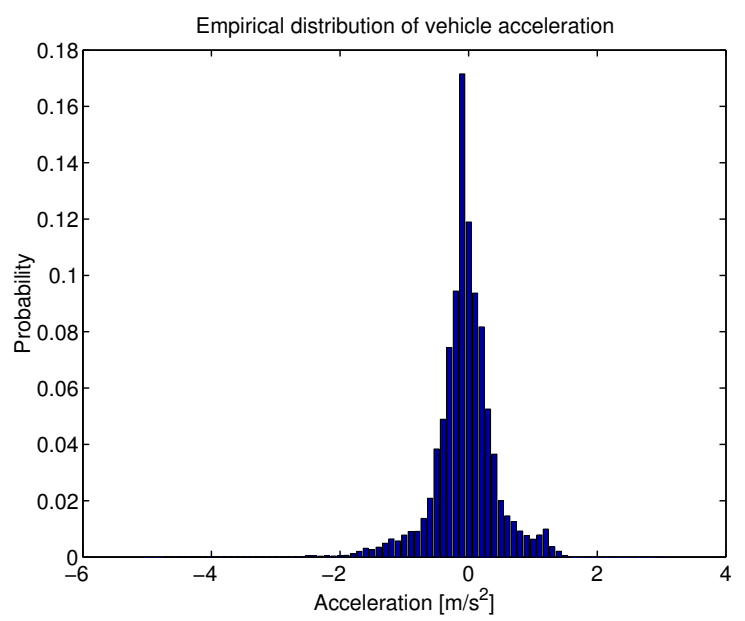

Fig. 1. Acceleration histogram of a vehicle driven in urban traffic for 45 minutes.

to switch between different distributions depending on the driving situation. In urban traffic one distribution is used, on highway another one, etc. This leads to a mode dependent distribution $p_{w}(w ; \xi)$. Here the mode can be determined from the host vehicle speed, it may depend on the state vector, external information from navigation and telematics systems etc. A simple approach is to use one mode when $v<70 \mathrm{~km} / \mathrm{h}$ and another one for $v>70 \mathrm{~km} / \mathrm{h}$.

\section{Measurement noise model}

The measurement equation for the radar involves range, range rate and relative angle measurements to the vehicle ahead. Similarly to the above, the range error distribution can be modeled. A common model is to assume Gaussian noise. However, we will extend this to a more general case. An experiment is performed by towing a radar equipped vehicle, which can then measure the range to the towing vehicle under driving conditions with an almost constant range. Data is collected using an FM-CW radar at $40 \mathrm{~m}$ and constant speed $60 \mathrm{~km} / \mathrm{h}$ on a Volvo S80 sedan. The range data is correlated, probably due to some oscillations in the rope and from internal filters in the receiver. A second order state space model is fitted to data using the $n 4 \mathrm{sid}$ method, [5], [6], in order to produce de-correlated range data. This pdf range error is presented in Fig 2 together with a Gaussian approximation.

The measurement noise from the experiment is collected under nearly ideal circumstances. For instance, the data was collected from behind, i.e., no aspect angle to the car, and on a smooth test track. In many cases due to road curvatures, uneven road surfaces and lateral movement the measurement range distribution might be different. Also, the exact reflection point is uncertain since the azimuth angle is not that exact, so for medium distances, the main reflection

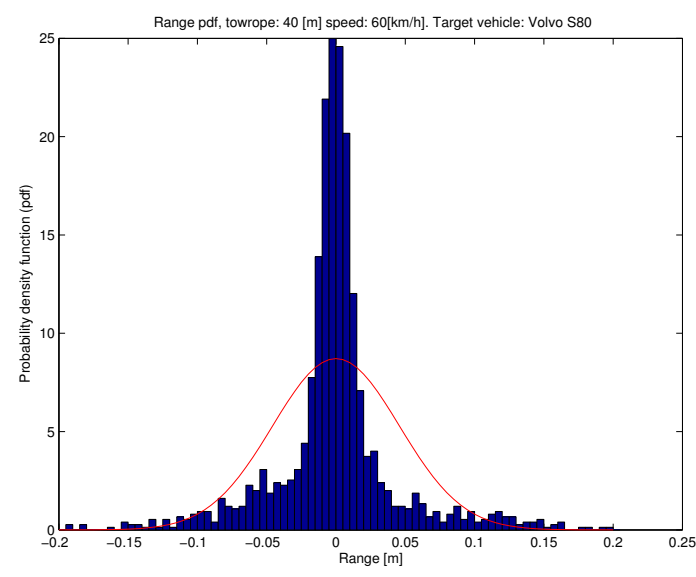

Fig. 2. Measurement range noise pdf around the nominal range $r=$ $40 \mathrm{~m}$ for the target vehicle Volvo S80 sedan and an equivalent Gaussian approximation.

point may be located on different parts of the car. Hence, there are indications that the measurement noise pdf has a larger variance and different shape. A bi-modal Gaussian pdf example is presented in Fig 3. This measurement noise model is motivated by modeling the vehicle by two point reflectors, which is a simplified way of describing both multi-path propagation and complex reflection geometry. The exact appearance of the pdf will vary with many factors such as target vehicle, sensor and traffic situation. Here we only test a bi-modal distribution with highly separated peaks. This is to cover many situations with different aspect angles, where the density is due to multi-path propagation, and multiple reflections points for instance in the rear-end, wheel housing and rear-view mirror. More measurements and experiments are needed to establish the empirical pdf for different driving situations, so the proposed density should just be an example of non-Gaussian pdf influence. In the simulation study we will compare different assumptions of the pdf.

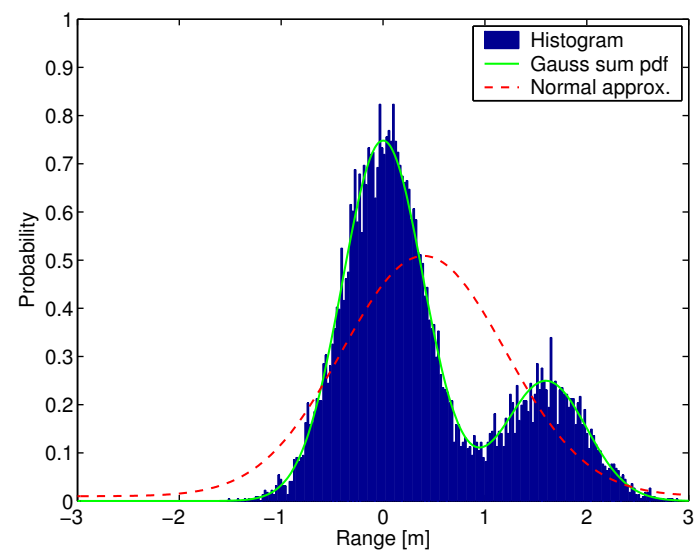

Fig. 3. Bimodal range measurement noise and Gaussian approximation of range density $p_{e}=0.75 \mathcal{N}\left(0,0.4^{2}\right)+0.25 \mathcal{N}\left(1.6,0.4^{2}\right)$. 

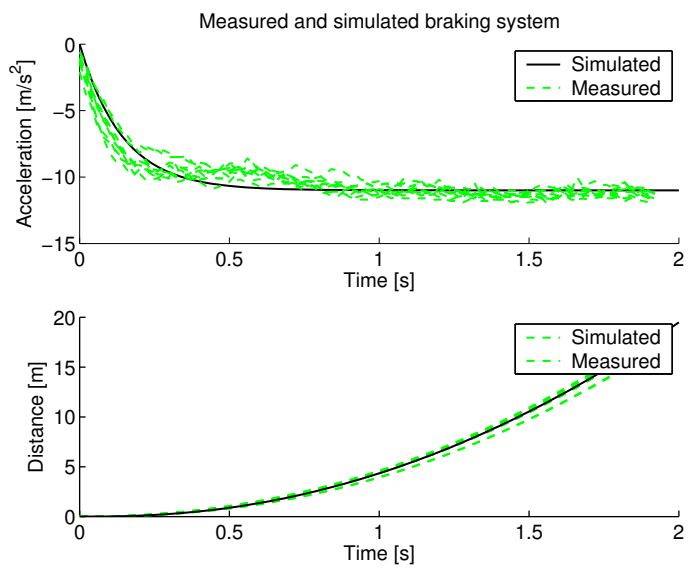

Fig. 4. Data from a hard braking maneuver, $110-0 \mathrm{~km} / \mathrm{h}$ for a Volvo V70.

\section{Statistical Decision MAKING}

Statistical decision making requires an estimate of the distribution of $x_{t}$ from noisy measurements $y_{t}$. The idea is for instance to calculate the probability for impact for each time. More on this for automotive application can be found in [1].

\section{A. Hypothesis testing}

A general model-based statistical decision rule based on some criterion, $g\left(x_{t}\right)$, takes the form

$$
\operatorname{Pr}\left\{g\left(x_{t}\right)>0\right\}>1-\alpha,
$$

where $\alpha$ determines the confidence level. Hence, in (2) one has to compute some integrals over the state-dimension to calculate the probability, given that we know the probability density function or an estimate thereof. Note that this is a more flexible approach than a simple rule like $g\left(\hat{x}_{t}\right)>0$, since in (2) we use the complete a posteriori distribution of the state vector and not only the estimate $\hat{x}_{t}$.

How successful the rule (2) is in mitigating a collision depends on the chosen rule, but also on how accurately the estimated pdf reflects the true distribution. Almost always an analytical expression of the probability is hard or impossible to compute. One way is to use a numerical integration or more preferrable a stochastic integration to compute an approximation. Here, one takes a large number of samples $x_{t}^{(i)}, i=1,2, \ldots, N$ from the distribution $p\left(x_{t} \mid y_{0}, \ldots, y_{t}\right)$, and then

$$
\operatorname{Pr}\left\{g\left(x_{t}\right)>0\right\} \approx \frac{\# g\left(x_{t}^{(i)}\right)>0}{N} .
$$

For the EKF a stochastic integration can be applied by drawing samples from the Gaussian distribution around the estimate $\hat{x}_{t}$. This procedure is much simpler when the particle filter is used, since the samples, $x_{t}^{(i)}$, exist internally in the algorithm. One can simply count the number of particles for which the inequality is satisfied. This fact decreases the gap in computational burden between the EKF and particle filter approaches.

\section{B. The $C M b B$ decision rule}

In this paper we will study a $\mathrm{CMbB}$ system, which performs maximum braking when a collision is becoming imminent, i.e., when a brake maneuver close to or exceeding the vehicles handling limits is needed to reach a zero speed at impact. We will introduce a very simple brake criteria to test the decision idea. We do this by calculating the required acceleration to obtain a zero velocity at a possible impact. Suppose at time $t=t_{0}$ the relative position and speed to the object ahead is given by $p_{0}$ and $v_{0}$. Denote the host vehicle acceleration by $u_{t}=a_{\text {host }}$ (input signal) and the target vehicle acceleration by $a_{o b j}$. We consider both of these accelerations to be constant from time $t>t_{0}$ and denote the relative acceleration by $a=a_{\text {host }}-a_{o b j}$. In the following analysis an ideal brake system is used for simplicity. For a high relative velocity the error of neglecting brake system characteristics can be compensated by adjusting the threshold in (7). The collision speed, $v^{c}$ at the collision time $t^{c}$, can be calculated as $v^{c}=v_{0}+a \cdot\left(t^{c}-t_{0}\right)$. The relative time to zero collision speed, $v^{c}=0$, is hence

$$
t^{z}=t^{c}-t_{0}=-\frac{v_{0}}{a} \text {. }
$$

For a simple motion model the zero velocity impact can be obtained using the relative acceleration solved from

$$
0=p_{0}+v(t) \cdot\left(t-t_{0}\right)+\frac{a \cdot\left(t-t_{0}\right)^{2}}{2},
$$

using (4). Hence, one obtains $a=-\frac{v_{0}^{2}}{2 p_{0}}$, which yield the host required acceleration

$$
a_{\text {host }}=a_{o b j}-a=a_{o b j}-\frac{v_{0}^{2}}{2 p_{0}} .
$$

For constant velocity case, i.e., $a_{o b j}=0$, the risk metric simplifies further and the hypothesis test will be according to

$$
\operatorname{Pr}\left\{-\frac{v_{0}^{2}}{2 p_{0}}<a_{t h}\right\}>1-\alpha,
$$

where $a_{t h}$ is the acceleration threshold for when we consider an accident to be imminent.

\section{Brake system model}

To calculate the collision speed the brake system characteristics need to be known. A complete brake system model is very complex. However looking at overall vehicle performance a simple yet accurate description can be obtained using a first order system for the acceleration. Fig 4 shows measurement data from 10 brake maneuvers with a Volvo V70 and a first order approximation The measurements were performed on warm and dry asphalt. For the simulated data a first order system was used with transfer function

$$
G_{\text {brake }}(s)=\frac{k_{1}}{s / k_{2}+1},
$$

with $k_{1}=11$ and $k_{2}=7$ chosen such that the system rise time is $0.3 \mathrm{~s}$ and the stationary value is $11 \mathrm{~m} / \mathrm{s}^{2}$, Comparing 
traveled distance after 2 seconds we find that the difference is less than $1 \mathrm{~m}$ which is sufficient for our purposes. More information on typical brake system behavior can be found in [7].

\section{BAYESIAN ESTIMATION}

Consider the state-space model

$$
\begin{aligned}
x_{t+1} & =f\left(x_{t}, u_{t}, w_{t}\right), \\
y_{t} & =h\left(x_{t}\right)+e_{t},
\end{aligned}
$$

where $x_{t} \in \mathbb{R}^{n}$ denotes the state of the system, $u_{t}$ the input signal and $y_{t}$ the observation at time $t$. The process noise $w_{t}$ and measurement noise $e_{t}$ are assumed independent with densities $p_{w_{t}}$ and $p_{e_{t}}$ respectively. Let $\mathbb{Y}_{t}=\left\{y_{i}\right\}_{i=1}^{t}$ be the set of observations until present time.

The Bayesian estimation problem is given by, [8],

$$
\begin{aligned}
p\left(x_{t+1} \mid \mathbb{Y}_{t}\right) & =\int_{\mathbb{R}^{n}} p\left(x_{t+1} \mid x_{t}\right) p\left(x_{t} \mid \mathbb{Y}_{t}\right) d x_{t}, \\
p\left(x_{t} \mid \mathbb{Y}_{t}\right) & =\frac{p\left(y_{t} \mid x_{t}\right) p\left(x_{t} \mid \mathbb{Y}_{t-1}\right)}{p\left(y_{t} \mid \mathbb{Y}_{t-1}\right)},
\end{aligned}
$$

where $p\left(x_{t+1} \mid \mathbb{Y}_{t}\right)$ is the prediction density and $p\left(x_{t} \mid \mathbb{Y}_{t}\right)$ the filtering density. The problem is is in general not analytically solvable.

\section{A. Particle filter}

To solve the non-tractable Bayesian estimation problem in an on-line application without using linearization or Gaussian assumptions, sequential Monte Carlo methods, or particle filters, could be used. Here only a brief description of the theory is given. For more details we refer to [9], [4], [10], [11]. The particle filter method provides an approximative Bayesian solution to (10) by approximating the probability density $p\left(x_{t} \mid \mathbb{Y}_{t}\right)$ by a large set of $N$ particles $\left\{x_{t}^{(i)}\right\}_{i=1}^{N}$, where each particle has an assigned relative weight, $\gamma_{t}^{(i)}$, such that all weights sum to unity. The location and weight of each particle reflect the value of the density in the region of the state space. The likelihood $p\left(y_{t} \mid x_{t}\right)$ is calculated from (9) yielding

$$
\gamma_{t}=p\left(y_{t} \mid x_{t}\right)=p_{e_{t}}\left(y_{t}-h\left(x_{t}\right)\right) .
$$

By introducing a resampling step as in [10] problems with divergence can be handled. This is referred to as sampling importance resampling (SIR), and is summarized in Alg 1.

Alg 1 (Sampling Importance Resampling (SIR)):

1. Generate $N$ samples $\left\{x_{0}^{(i)}\right\}_{i=1}^{N}$ from $p\left(x_{0}\right)$.

2. Compute $\gamma_{t}^{(i)}=p_{e}\left(y_{t} \mid x_{t}^{(i)}\right)$ and normalize, i.e., $\bar{\gamma}_{t}^{(i)}=\gamma_{t}^{(i)} / \sum_{j=1}^{N} \gamma_{t}^{(j)}, i=1, \ldots, N$.

3. Generate a new set $\left\{x_{t}^{(i \star)}\right\}_{i=1}^{N}$ by resampling with replacement $N$ times from $\left\{x_{t}^{(i)}\right\}_{i=1}^{N}$, with probability $\bar{\gamma}_{t}^{(j)}=\operatorname{Pr}\left\{x_{t}^{(i \star)}=x_{t}^{(j)}\right\}$.

4. Prediction: $x_{t+1}^{(i)}=f\left(x_{t}^{(i \star)}, u_{t}, w_{t}^{(i)}\right), i=1, \ldots, N$ using different noise realizations $w_{t}^{(i)}$.

5. Increase $t$ and iterate to step 2 .
The decision criterion (7) can easily be evaluated for the particle filter since the particles, $x_{t}^{(i)}$ reflects the location and distribution of the states. Hence, the particle filter is well suited for statistical decision making.

\section{B. Extended Kalman filter}

The Bayesian recursions in Section IV do not in general have an analytical solution. For the special case of linear dynamics, linear measurements and Gaussian noise there exist a solution, which is retrieved by the Kalman filter, [12]. For many non-linear problems the noise assumptions are such that a linearized solution will be a good approximation. This is the idea behind the EKF, [13], where the model is linearized around the previous estimate. The time- and measurement update for the EKF are give by

$$
\begin{aligned}
& \left\{\begin{array}{l}
\hat{x}_{t+1 \mid t}=f\left(\hat{x}_{t \mid t}, u_{t}\right), \\
P_{t+1 \mid t}=F_{t} P_{t \mid t} F_{t}^{T}+G_{t} Q_{t} G_{t}^{T},
\end{array}\right. \\
& \left\{\begin{array}{l}
\hat{x}_{t \mid t}=\hat{x}_{t \mid t-1}+K_{t}\left(y_{t}-h\left(\hat{x}_{t \mid t-1}\right)\right), \\
P_{t \mid t}=P_{t \mid t-1}-K_{t} H_{t} P_{t \mid t-1}, \\
K_{t}=P_{t \mid t-1} H_{t}^{T}\left(H_{t} P_{t \mid t-1} H_{t}^{T}+R_{t}\right)^{-1},
\end{array}\right.
\end{aligned}
$$

with linearized matrices and covariances

$$
\begin{aligned}
& F_{t}=\left.\nabla_{x} f\left(x_{t}\right)\right|_{x_{t}=\hat{x}_{t \mid t-1}}, H_{t}=\left.\nabla_{x} h\left(x_{t}\right)\right|_{x_{t}=\hat{x}_{t \mid t-1}}, \\
& Q_{t}=\operatorname{Cov}\left(w_{t}\right), R_{t}=\operatorname{Cov}\left(e_{t}\right) .
\end{aligned}
$$

When a multi-modal Gaussian measurement pdf is used

$$
e_{t} \in \sum_{i=1}^{M} p_{i} \mathcal{N}\left(\mu_{i}, \sigma_{i}^{2}\right),
$$

where $\mathcal{N}\left(\mu, \sigma^{2}\right)$ denotes a Gaussian density with mean $(\mu)$ and covariance $\left(\sigma^{2}\right)$, the measurement update equation must be modified as

$$
\hat{x}_{t \mid t}=\hat{x}_{t \mid t-1}+K_{t}\left(y_{t}-h\left(\hat{x}_{t \mid t-1}\right)-\bar{m}\right),
$$

where $\bar{m}=\sum_{i=1}^{M} p_{i} \mu_{i}$. If the measurement pdf is not know analytically, the approximation using a single Gaussian pdf introduces a bias (since $\bar{m}=0$ ).

The decision criterion (7) is not analytically solvable when the state variables are considered Gaussian. By sampling the position and velocity distribution around the estimates a Monte Carlo integration technique can solve the decision criterion. Hence, part of the computational burden present in the particle filter is introduce in this step for the EKF method.

\section{Simulations And Tests}

In a simulation study we compare the traditionally EKF tracking filter with the particle filter using the previously described decision rule for braking. We also test the realtime performance in a hardware collision avoidance test platform. 


\section{A. Model}

To simplify analysis of the system performance for different noise distributions, we consider a single scenario. The scenario to be studied is one where the target object is not moving and the vehicle with the CMbB system is approaching with constant velocity $(60 \mathrm{~km} / \mathrm{h})$.

Consider the state vector with relative position $p$ and velocity $v$ for the $x$ - and $y$-direction, where the sensor measures relative distance, azimuth and relative speed.

$$
x_{t}=\left(\begin{array}{llll}
p_{x} & p_{y} & v_{x} & v_{y}
\end{array}\right)^{T} .
$$

The tracking model with sample time $T$ is then

$$
\begin{gathered}
x_{t+1}=F x_{t}+G w_{t} \\
y_{t}=h\left(x_{t}\right)+e_{t}, \\
F=\left(\begin{array}{cccc}
1 & 0 & T & 0 \\
0 & 1 & 0 & T \\
0 & 0 & 1 & 0 \\
0 & 0 & 0 & 1
\end{array}\right), G=\left(\begin{array}{cc}
T^{2} / 2 & 0 \\
0 & T^{2} / 2 \\
T & 0 \\
0 & T
\end{array}\right), \\
h\left(x_{t}\right)=\left(\begin{array}{c}
r \\
\varphi \\
\dot{r}
\end{array}\right)=\left(\begin{array}{c}
\sqrt{p_{x}^{2}+p_{y}^{2}} \\
\arctan \left(p_{y} / p_{x}\right) \\
\frac{1}{\sqrt{p_{x}^{2}+p_{y}^{2}}}\left(p_{x} v_{x}+p_{y} v_{y}\right)
\end{array}\right) .
\end{gathered}
$$

The process noise, $w_{t}$, and measurement noise, $e_{t}$, are assumed to be independent white noise.

\section{B. Scenario}

We consider two main models for the measurement sensor. In the first we use the measured range distribution from Fig 2, based on measurements collected from experimental data on a Volvo S80. We conclude that the range density is narrow and not as bimodal as expected. This is probably due to the ideal circumstances in the experiment as described in Section II-D. We are also interested in other driving situations. Hence the second model of the measurement range noise is a bi-modal Gaussian, i.e.,

$$
e_{t} \in p_{1} \mathcal{N}\left(\mu_{1}, \sigma_{1}^{2}\right)+p_{2} \mathcal{N}\left(\mu_{2}, \sigma_{2}^{2}\right)
$$

where $\mathcal{N}\left(\mu, \sigma^{2}\right)$ denotes a Gaussian density with mean $(\mu)$ and covariance $\left(\sigma^{2}\right)$. A sample of $e_{t}$ belongs to one of the distributions, with probability $p_{i}$. The values are given in Table I. Here $p_{1}=0.75$ and $p_{2}=0.25$ since the rear-end of the vehicle is assumed to have a larger radar cross section. The motivation for studying the bi-modal distribution is discussed in Section II-D. Here two different parameterizations are presented to investigate the influence.

The range rate and azimuth were found to to be well approximated by Gaussian distributions, with $\sigma_{\dot{r}}=0.2$ and $\sigma_{\varphi}=0.01$.

We use a longitudinal acceleration process noise, $Q \approx$ $0.5 / T$, to model driving behavior and model imperfections.

A simple brake system model according to (8) is used in the simulations, with parameters $k_{1}$ and $k_{2}$ chosen to give a brake system rise-time of $300 \mathrm{~ms}$ and a maximum deceleration of $9.8 \mathrm{~m} / \mathrm{s}^{2}$. The somewhat lower maximum deceleration (compared to section II) is due to the fact that the test track exhibit optimal frictional conditions.

The brake decision is based on a hypothesis test on the expected required acceleration according to (7). We have chosen the acceleration threshold to $a_{t h}=-8 \mathrm{~m} / \mathrm{s}^{2}$ and the confidence level to $\alpha=0.05$. For the SIR approach the hypothesis is evaluated for each particle.

We perform 1000 Monte Carlo (MC) simulations for both SIR and EKF. In the particle filter we use $N=5000$ particles and the same amount of samples in the stochastic integration for the EKF.

\section{Simulation results}

The different simulations from the Monte Carlo study are summarized in Table I together with tracking and CMbB system performance. Three different cases were considered. In case I and II two different bi-modal Gaussian range distributions were used and in case III the empirical range distribution from Fig 2 were used. All other values were the same for all the simulation cases. For non-Gaussian range distributions such as the bimodal-Gaussian proposed for the measurement noise, the SIR method increases estimation performance (position RMSE) slightly. The mean difference in collision speed is basically not affected, however the EKF has a somewhat larger collision speed variance, but probably insignificant. The result is under the assumptions of quite accurate range rate and azimuth measurements and a high measurement update rate, $f=1 / T=20 \mathrm{~Hz}$. Hence, for systems that do not measure the relative speed more significant differences are expected.

\section{Hardware and real-time issues}

In the simulations presented in Section V-A the entire algorithm was implemented in standard MATLAB code. However, the ultimate goal with the particle filter based approach is to incorporate the algorithm and test the idea in an on-line application in a collision avoidance system. In Fig 5 the test system is shown mounted inside the test vehicle. The test platform uses dSpace hardware (equipped

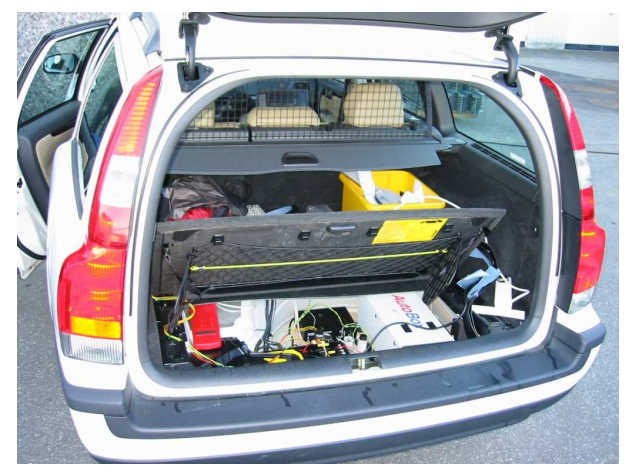

Fig. 5. The collision avoidance hardware in the test vehicle.

with a $1 \mathrm{GHz}$ power $\mathrm{PC}$ ) together with Simulink and 


\begin{tabular}{|c|c|c|c|c|c|c|}
\hline Case & Filter & Range pdf $p_{e_{t}}$ & RMSE (pos) [m] & RMSE (vel) [m/s] & Coll.speed [m/s] & $\sigma$ Coll.speed [m/s] \\
\hline \hline I & SIR & $0.75 \mathcal{N}\left(0,0.4^{2}\right)+0.25 \mathcal{N}\left(1.6,0.4^{2}\right)$ & 0.11 & 0.29 & -6.59 & 0.51 \\
\hline I & EKF & $0.75 \mathcal{N}\left(0,0.4^{2}\right)+0.25 \mathcal{N}\left(1.6,0.4^{2}\right)$ & 0.16 & 0.29 & -6.61 & 0.53 \\
\hline \hline II & SIR & $0.75 \mathcal{N}\left(0,0.2^{2}\right)+0.25 \mathcal{N}\left(1,0.4^{2}\right)$ & 0.08 & 0.29 & -6.56 & 0.49 \\
\hline II & EKF & $0.75 \mathcal{N}\left(0,0.2^{2}\right)+0.25 \mathcal{N}\left(1,0.4^{2}\right)$ & 0.11 & 0.29 & -6.56 & 0.51 \\
\hline \hline III & SIR & Empirical (Fig 2) & 0.06 & 0.29 & -6.52 & 0.47 \\
\hline III & EKF & Empirical (Fig 2) & 0.06 & 0.29 & -6.52 & 0.47 \\
\hline
\end{tabular}

RESUlts FROM 1000 MONTE CARLO SIMULATIONS AND DIFFERENT CASES.

Real-Time Workshop (RTW). Hence, the algorithm must be written in C-code and MATLAB/MEX functions for Simulink. The entire environment is then compiled using the RTW-compiler to the dedicated hardware platform. In Fig 6 the Simulink diagram is shown for the underlying MEX C-code functions, i.e., the particle filter and the decision making algorithm. These are incorporated in the testbed using the RTW-compiler. The entire algorithm runs faster then real-time on the dedicated hardware, with $T=0.05 \mathrm{~s}$ and $N=5000$ particles.

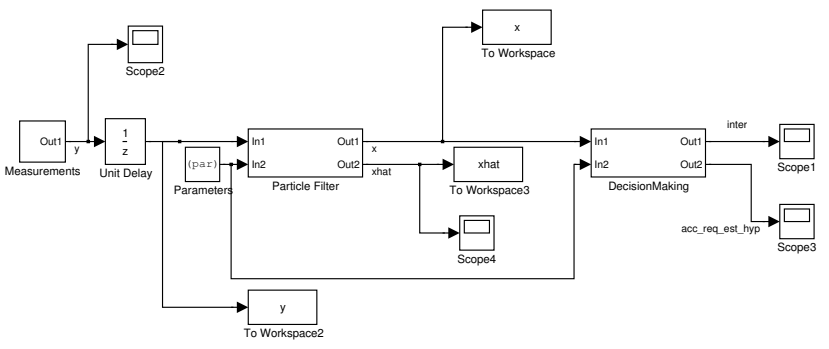

Fig. 6. MATLAB Simulink diagram of the CMbBS model used to generate RTW-code for the dedicated hardware.

\section{CONCLUSIONS}

We have implemented a decision rule in a CMbB system for late braking using a hypothesis test based on estimates of the relative longitudinal dynamics. Both an EKF and a particle filter were evaluated for different noise assumptions. For non-Gaussian range distributions such as the bimodalGaussian proposed for the measurement noise, the SIR method increases estimation performance slightly. However, the range error is in the order of decimeters for all tested scenarios, so the mean difference in collision speed is basically not affected. The EKF has a somewhat larger collision speed variance, but probably insignificant. The result is under the assumption of quite accurate range rate and azimuth measurements, and a moderate process noise. Hence, a greater difference might occur if for instance the range rate is not measured or if more maneuverability, i.e., larger process noise is considered.

The computational cost of the EKF method is somewhat smaller than for the SIR, but rather close. This is due to the fact that the EKF uses a stochastic integration to calculate the probability of collision. So for a decision rule like (2) a particle filter approach might be preferable. It should also be kept in mind that the scenario studied here is very simple (from a tracking sense), for more complex scenarios with maneuvering target and tracking platform the difference between the methods may be larger. Also the particle filter method is more flexible, so if a more complex state-space model is used, linearization errors in the dynamical model can be avoided.

In the simulation study the simulations for both the SIR and EKF approach were run faster than real-time on an ordinary desk-top computer. The particle filter was also implemented in the Volvo testbed hardware (used in vehicle tests) using the MATLAB Simulink RTW compiler. The tracking filter and decision algorithm executed faster than the real-time constraint, $T=0.05$, using $N=5000$ particles for the tracking model described earlier.

The authors would like to thank Volvo Car, Sweden, especially Fredrik Lundholm and Lena Westervall for providing measurement data and a hardware platform to test the algorithm on.

\section{REFERENCES}

[1] J. Jansson, Tracking and Decision Making for Automotive Collision Avoidance. Department of Electrical Engineering, Linköping University, Sweden: Licentiate Thesis no. 965, September 2002.

[2] B. Zhu, "Potential effects on accidents from forward collision warning/avoidance system," ITN, Linköping University, S-581 83 Linköping, Sweden, Master Thesis LITH-ITN-EX-150-SE, 2001.

[3] J. Treat, N. McDonald, D. Shinar, R. Hume, R. Mayer, R. Stansifer, and N. Castellan, "Tri-level study of the causes of traffic accidents," NHTSA, Tech. Rep., 1977.

[4] A. Doucet, N. de Freitas, and N. Gordon, Eds., Sequential Monte Carlo Methods in Practice. Springer Verlag, 2001.

[5] L. Ljung, System Identification, Theory for the User, 2nd ed. Englewood Cliffs, New Jersey: Prentice Hall, 1999.

[6] - System Identification Toolbox. The MathWorks, Inc.

[7] D.-I. U. Adler, Automotive Handbook, 5th ed. Robert Bosch GmbH, 2000, no. ISBN: 0-7680-0669-4.

[8] A. Jazwinski, Stochastic processes and filtering theory, ser. Mathematics in Science and Engineering. Academic Press, 1970, vol. 64.

[9] N. Bergman, "Recursive bayesian estimation: Navigation and tracking applications," Ph.D. dissertation, Linköping University, 1999, dissertations No. 579.

[10] N. Gordon, D. Salmond, and A. Smith, "A novel approach to nonlinear/non-Gaussian Bayesian state estimation," in IEE Proceedings on Radar and Signal Processing, vol. 140, 1993, pp. 107-113.

[11] R. Karlsson, Simulation Based Methods for Target Tracking. Department of Electrical Engineering, Linköping University, Sweden: Licentiate Thesis no. 930, Feb 2002.

[12] R. E. Kalman, "A new approach to linear filtering and prediction problems," Trans. AMSE, J. Basic Engineering, vol. 82, pp. 35-45, 1960.

[13] B. Anderson and J. Moore, Optimal Filtering. Englewood Cliffs, NJ: Prentice Hall, 1979. 